\title{
RELATIONSHIP OF THE BERNARDI INTEGRAL OPERATOR WITH CERTAIN CLASSES OF ANALYTIC FUNCTIONS
}

\author{
MUHAMMAD ARIF, MOHSAN RAZA, MIRAJ UL HAQ, \\ AND GAUTAM SRIVASTAVA
}

Received 29 November, 2019

\begin{abstract}
In this article, we find a condition on $\alpha$ so that if $1+\alpha z \frac{z f^{\prime}(z)}{f(z)} \prec \frac{1+A z}{1+B z}$, then $\frac{z \mathcal{F}^{\prime}(z)}{\mathcal{F}(z)} \prec$ $1+\sin (z)$, where

$$
\mathcal{F}(z)=\frac{\gamma+1}{z^{\beta}} \int_{0}^{z} t^{\gamma-1} f(t) d t,
$$
\end{abstract}

is the well-known Bernardi integral operator. We also study the case $\frac{z \mathcal{F}^{\prime}(z)}{\mathcal{F}(z)} \prec \cos (z)$. Some similar implications are also discussed for both functions.

2010 Mathematics Subject Classification: 30C45; 30C50

Keywords: Bernardi integral operator, Janowski functions, trigonometric functions

\section{INTRODUCTION AND BASIC NOTIONS}

We recall here some basic notions and concepts of geometric function theory which are essential for clarity and understandings of the upcoming work. We start with the symbol $\mathcal{A}$ which represents the class of analytic functions in $\mathcal{D}=\{z:|z|<1\}$ and any function $f$ in $\mathcal{A}$ satisfies the conditions $f(0)=f^{\prime}(0)-1=0$. Also let $\mathcal{S}$ denote a subclass of $\mathcal{A}$ which contains univalent functions in $\mathcal{D}$. The notion of subordinations between analytic functions is defined as; If $f$ and $g$ are analytic functions, then $f$ is subordinated by $g$, if there exists a Schwarz function $w$ with the properties $w(0)=0$ and $|w(z)|<|z|$ such that $f(z)=g(w(z))$. Further, if the function $g$ is univalent in $\mathcal{D}$, then we have:

$$
f(z) \prec g(z) \Leftrightarrow f(0)=g(0),
$$

and $f(\mathcal{D}) \subset g(\mathcal{D})$. Moreover, let $\mathcal{P}[A, B]$ denote a family of analytic functions $p$ in $\mathcal{D}$ with $p(0)=1$ and satisfy

$$
p(z) \prec \frac{1+A z}{1+B z}, \quad-1 \leq B<A \leq 1 .
$$

Corresponding author: Mohsan Raza. 
Geometrically, a function $p \in \mathcal{P}[A, B]$, if and only if $p(0)=1$ and $p(\mathcal{D}) \subset \Omega[A, B]$, where circular domain $\Omega[A, B]$ is defined as:

$$
\Omega[A, B]=\left\{\begin{array}{lll}
\left\{\omega:\left|\omega-\frac{1-A B}{1-B^{2}}\right|<\frac{A-B}{1-B^{2}}\right\} & \text { for } B \neq-1, \\
\{\omega: \mathfrak{R e}\{\omega\}>(1-A) / 2\} & \text { for } \quad B=-1 .
\end{array}\right.
$$

The domain $\Omega[A, B], B \neq-1$, represents an open circular disk centered on the real axis with diameter end points $D_{1}=\frac{1-A}{1-B}$ and $D_{2}=\frac{1+A}{1+B}$ with $0<D_{1}<1<D_{2}$. This work was introduced by Janowski [9] and he further defined the class $\mathcal{S}^{*}[A, B]$ of Janowski starlike functions as:

$$
\mathcal{S}^{*}[A, B]=\left\{f: f \in \mathcal{A} \text { and } \frac{z f^{\prime}(z)}{f(z)} \in \mathcal{P}[A, B],(z \in \mathcal{D})\right\} .
$$

We see that the classes $\mathcal{P}[1,-1]$ and $\mathcal{S}^{*}[1,-1]$ coincides with the well-known classes $\mathcal{P}$ of functions with positive real part and $\mathcal{S}^{*}$ of starlike functions respectively. Many researchers introduced and studied various subclasses of analytic functions connected with different image domains, see the work of Cho et al. [5], Dziok et al. [7], Kumar and Ravichandran [12], Mediratta et al. [15], Sokół and Stankiewicz [22], Raina and Sokół [16], Kanas and Răducanu [10], Sharma et al. [17], see also [1,11,18-20,25] and the references therein.

Recently, Cho et al. [6] have studied a class of starlike functions connected with sine function and is defined as:

$$
\mathcal{S}_{s}^{*}=\left\{f: f \in \mathcal{A} \text { and } \frac{z f^{\prime}(z)}{f(z)} \prec 1+\sin (z), \quad(z \in \mathcal{D})\right\} .
$$

Analogous to the class $\mathcal{S}_{s}^{*}$, Bano and Raza [3] introduced the class $\mathcal{S}_{c}^{*}$ of starlike functions associated with cosine functions which is given by:

$$
\mathcal{S}_{c}^{*}=\left\{f: f \in \mathcal{A} \text { and } \frac{z f^{\prime}(z)}{f(z)} \prec \cos (z), \quad(z \in \mathcal{D})\right\} .
$$

Integral and differential operators are very useful and are of great importance in geometric function theory, specially in univalent function theory. Certain differential and integral operators have been introduced by using convolution of certain analytic functions. It is observed that this formalism brings an ease in further mathematical exploration and also helps to understand the geometric properties of analytic and univalent functions. Their study can be traced back to 1916 by Alexander. Later, Libera and Bernardi introduced certain integral operators to study the classes of starlike, convex and close-to-convex functions. Recently, many researchers have shown great interest in studying some properties of integral and differential operators. Srivastava et al. [24] applied the fractional $q$-calculus operator to define and study a new class of analytic functions with complex order. Mahmood et al. [13] considered oneparameter families of integral operators and, studied the classes of uniformly starlike and uniformly convex functions with respect to symmetric points. Mehmood et al. 
[14] analyzed certain classes of analytic functions defined by using $q$-integral operators. Arif et al. [2] introduced a class of multivalent functions by using $q$-analogue of the Ruscheweyh type operator. Sim et al. [21] investigated argument properties of analytic functions defined by the Srivastava-Attiya Operator. Srivastava et al. [26] found Faber polynomial coefficients for bi-univalent functions defined by using fractional derivative operator. Recently, Srivastava [23] gave brief survey of operators of basic (or $q$-) calculus and fractional $q$-calculus and their applications in geometric function theory of complex analysis.

Motivated by the above work, we study the inclusion of Bernardi integral operator in the classes of starlike functions associated with sine and cosine functions in $\mathcal{D}$. The Bernardi [4] integral operator is defined as:

$$
\mathcal{F}(z)=\frac{\gamma+1}{z^{\beta}} \int_{0}^{z} t^{\gamma-1} f(t) d t, \gamma \geq 0 .
$$

To prove our main results we need the following lemma.

Lemma 1 ([8]). Let $w$ be a non-constant analytic function in $\mathcal{D}$ with $w(0)=0$. If

$$
\left|w\left(z_{0}\right)\right|=\max \left\{|w(z)|, \quad|z| \leq\left|z_{0}\right|\right\}, \quad z \in \mathcal{D},
$$

then there exists a real number $m(m \geq 1)$ such that $z_{0} w^{\prime}\left(z_{0}\right)=m w\left(z_{0}\right)$.

\section{BERNARDI'S INTEGRAL OPERATOR ASSOCIATED WITH SINE FUNCTION}

Theorem 1. Assume that

$$
|\alpha| \geq \frac{(A-B)(1+\gamma+\sin h(1))}{(1-|B|) \cos (1)-(1+|B|)(1+\gamma+\sin h(1))(1+\sin h(1))} .
$$

If

$$
1+\alpha z\left(\frac{z f^{\prime}(z)}{f(z)}\right) \prec \frac{1+A z}{1+B z},-1 \leq B<A \leq 1,
$$

then

$$
\frac{z \mathcal{F}^{\prime}(z)}{\mathcal{F}(z)} \prec 1+\sin (z)
$$

where $\mathcal{F}$ is the Bernardi integral operator defined in (1.3).

Proof. Let us define a function $w$ as

$$
w(z)=\arcsin \left(\frac{z \mathcal{F}^{\prime}(z)}{\mathcal{F}(z)}-1\right),
$$

where we have chosen the principle branches of the square root and logarithmic functions. Since arcsin functions is defined as

$$
\arcsin z=-i \log \left[i z+\left(1-z^{2}\right)^{1 / 2}\right],
$$


therefore $w$ is analytic in $\mathcal{D}$ with $w(0)=0$. To prove our result, we need only to show that $|w(z)|<1$ in $\mathcal{D}$. From $(2.3)$, we have

$$
\frac{z \mathcal{F}^{\prime}(z)}{\mathcal{F}(z)}=1+\sin (w(z)) .
$$

Logarithmic differentiation of above relation yields

$$
1+\frac{z \mathcal{F}^{\prime \prime}(z)}{\mathcal{F}^{\prime}(z)}-\frac{z \mathcal{F}^{\prime}(z)}{\mathcal{F}(z)}=\frac{z w^{\prime}(z) \cos (w(z))}{1+\sin (w(z))} .
$$

Differentiating (1.3), we obtain

$$
(\gamma+1) f(z)=z \mathcal{F}^{\prime}(z)+\gamma \mathcal{F}(z) .
$$

Differentiating (2.4) logarithmically, we have

$$
\begin{aligned}
\frac{z f^{\prime}(z)}{f(z)} & =\frac{z \mathcal{F}^{\prime}(z)}{\mathcal{F}(z)}\left\{\frac{1+\frac{z \mathcal{F}^{\prime \prime}(z)}{\mathcal{F}^{\prime}(z)}-\frac{z \mathcal{F}^{\prime}(z)}{\mathcal{F}(z)}}{\frac{z \mathcal{F}^{\prime}(z)}{\mathcal{F}(z)}+\gamma}+1\right\} \\
& =\frac{z w^{\prime}(z) \cos (w(z))+(1+\sin (w(z))+\gamma)(1+\sin (w(z)))}{(1+\sin (w(z))+\gamma)} .
\end{aligned}
$$

Now, we define a function

$$
p(z)=1+\alpha z\left(\frac{z f^{\prime}(z)}{f(z)}\right),
$$

where $p$ is analytic in $\mathcal{D}$ and $p(0)=1$. Also

$$
\left|\frac{p(z)-1}{A-B p(z)}\right|=\left|\frac{1}{\frac{(A-B)(1+\sin (w(z))+\gamma)}{\alpha z\left[z w^{\prime}(z) \cos (w(z))+(1+\sin (w(z))+\gamma)(1+\sin (w(z)))\right]}+B}\right| \text {. }
$$

Suppose that there exists a point $z_{0} \in \mathcal{D}$ such that

$$
\max _{|z| \leq\left|z_{0}\right|}|w(z)|=\left|w\left(z_{0}\right)\right|=1 .
$$

By using Lemma 1 , there exists a number $m \geq 1$ such that $z_{0} w^{\prime}\left(z_{0}\right)=m w\left(z_{0}\right)$. We also suppose that $w\left(z_{0}\right)=e^{i \theta}$. Then we have

$$
\begin{aligned}
\left|\frac{p\left(z_{0}\right)-1}{A-B p\left(z_{0}\right)}\right| & =\left|\frac{1}{\frac{(A-B)\left(1+\sin \left(w\left(z_{0}\right)\right)+\gamma\right)}{\alpha\left[z w^{\prime}\left(z_{0}\right) \cos \left(w\left(z_{0}\right)\right)+\left(1+\sin \left(w\left(z_{0}\right)\right)+\gamma\right)\left(1+\sin \left(w\left(z_{0}\right)\right)\right)\right]}+B}\right| \\
& =\left|\frac{1}{\frac{(A-B)\left(1+\sin \left(e^{i \theta}\right)+\gamma\right)}{\alpha\left[m e^{i \theta} \cos \left(e^{i \theta}\right)+\left(1+\sin \left(e^{i \theta}\right)+\gamma\right)\left(1+\sin \left(e^{i \theta}\right)\right)\right]}+B}\right| .
\end{aligned}
$$


Let $|z|=r,-\pi \leq \theta \leq \pi$. Then after some simplifications, we have

$$
\left|\sin \left(e^{i \theta}\right)\right|^{2}=\cos ^{2}(\cos \theta) \sin h^{2}(\sin \theta)+\sin ^{2}(\cos \theta) \cos h^{2}(\sin \theta)=\Psi(\theta) .
$$

A simple computation shows that the equation $\Psi^{\prime}(\theta)=0$ has five roots in $[-\pi, \pi]$ namely $0, \pm \pi, \pm \frac{\pi}{2}$. Since $\Psi^{\prime}(\theta)=\Psi^{\prime}(-\theta)$, it is sufficient to consider $\theta \in[0, \pi]$ and we see that

$$
\Psi^{\prime \prime}(0)=\Psi^{\prime \prime}(\pi) \approx 1.090702574 \text { and } \Psi^{\prime \prime}\left(\frac{\pi}{2}\right) \approx-1.626860410 .
$$

This implies that

$$
\begin{aligned}
& \max \{\Psi(\theta)\}=\Psi\left(\frac{\pi}{2}\right)=\sin h^{2}(1) \\
& \min \{\Psi(\theta)\}=\Psi(0)=\Psi(\pi)=\sin ^{2}(1)
\end{aligned}
$$

Also consider

$$
\left|\cos \left(e^{i \theta}\right)\right|^{2}=\cos ^{2}(\cos \theta) \cos h^{2}(\sin \theta)+\sin ^{2}(\cos \theta) \sin h^{2}(\sin \theta)=\phi(\theta) .
$$

Similarly, after simple calculations the equation $\phi^{\prime}(\theta)=0$ has five roots in $[-\pi, \pi]$ namely $0, \pm \pi, \pm \frac{\pi}{2}$. Since $\phi(\theta)=\phi(-\theta)$, it is sufficient to consider those roots which lies in $[0, \pi]$ and we see that

$$
\phi^{\prime \prime}(0)=\phi(\pi) \approx 2.909297427 \text { and } \phi^{\prime \prime}\left(\frac{\pi}{2}\right) \approx-5.626860410 .
$$

Therefore

$$
\begin{aligned}
& \max \{\phi(\theta)\}=\phi\left(\frac{\pi}{2}\right)=\cos h^{2}(1), \\
& \min \{\phi(\theta)\}=\phi(\pi)=\phi(0)=\cos ^{2}(1) .
\end{aligned}
$$

This implies that

$$
\cos (1) \leq\left|\cos \left(e^{i \theta}\right)\right| \leq \cosh (1) \text { and } \sin (1) \leq\left|\sin \left(e^{i \theta}\right)\right| \leq \sin h(1) .
$$

Now

$$
\begin{aligned}
& \left|\frac{p\left(z_{0}\right)-1}{A-B p\left(z_{0}\right)}\right|=\left|\frac{1}{\frac{(A-B)\left(1+\sin \left(e^{i \theta}\right)+\gamma\right)}{\alpha\left[m e^{i \theta} \cos \left(e^{i \theta}\right)+\left(1+\sin \left(e^{i \theta}\right)+\gamma\right)\left(1+\sin \left(e^{i \theta}\right)\right)\right]}+B}\right| \\
& \geq \frac{|\alpha|\left[\left|m e^{i \theta} \cos \left(e^{i \theta}\right)\right|-\left|\left(1+\sin \left(e^{i \theta}\right)+\gamma\right)\left(1+\sin \left(e^{i \theta}\right)\right)\right|\right]}{(A-B)\left|\left(1+\sin \left(e^{i \theta}\right)+\gamma\right)\right|+|\alpha B|\left[\left|m e^{i \theta} \cos \left(e^{i \theta}\right)\right|+\left|\left(1+\sin \left(e^{i \theta}\right)+\gamma\right)\left(1+\sin \left(e^{i \theta}\right)\right)\right|\right]} \\
& \geq \frac{|\alpha|[m \cos (1)-(1+\gamma+\sin h(1))(1+\sin h(1))]}{(A-B)(1+\gamma+\sin h(1))+|\alpha B|[m \cos (1)+(1+\gamma+\sin h(1))(1+\sin h(1))]} .
\end{aligned}
$$


Now let

$$
\Phi(m)=\frac{|\alpha|[m \cos (1)-(1+\gamma+\sin h(1))(1+\sin h(1))]}{(A-B)(1+\gamma+\sin h(1))+|\alpha B|[m \cos (1)+(1+\gamma+\sin h(1))(1+\sin h(1))]} .
$$

Then

$$
\begin{aligned}
& \Phi^{\prime}(m)= \\
& \frac{\left\{(A-B)(1+\gamma+\sin h(1))+2|\alpha|^{2}|B|[(1+\gamma+\sin h(1))(1+\sin h(1))]\right\}|\alpha| \cos (1)}{\{(A-B)(1+\gamma+\sin h(1))+|\alpha B|[m \cos (1)+(1+\gamma+\sin h(1))(1+\sin h(1))]\}^{2}}>0 .
\end{aligned}
$$

This shows that $\Phi$ is an increasing function and it has its minimum value at $m=1$ so

$$
\left|\frac{p\left(z_{0}\right)-1}{A-B p\left(z_{0}\right)}\right| \geq \frac{|\alpha|[\cos 1-(1+\gamma+\sin h(1))(1+\sin h(1))]}{(A-B)(1+\gamma+\sin h(1))+|\alpha B|[\cos 1+(1+\gamma+\sin h(1))(1+\sin h(1))]} \text {. }
$$

Now by (2.1), we have

$$
\left|\frac{p\left(z_{0}\right)-1}{A-B p\left(z_{0}\right)}\right| \geq 1
$$

a contradiction to the hypothesis. Hence we have the required result.

By choosing $A=1, B=0$, we have the following result.

Corollary 1. Let

$$
|\alpha| \geq \frac{(1+\gamma+\sin h(1))}{\cos (1)-(1+\gamma+\sin h(1))(1+\sin h(1))}
$$

If

$$
1+\alpha z\left(\frac{z f^{\prime}(z)}{f(z)}\right) \prec 1+z
$$

then

$$
\frac{z \mathcal{F}^{\prime}(z)}{\mathcal{F}(z)} \prec 1+\sin (z)
$$

By choosing $A=1, B=-1$, we have the following result.

Corollary 2. Let $|\alpha| \geq-0.363370517$ and

$$
1+\alpha z\left(\frac{z f^{\prime}(z)}{f(z)}\right) \prec \frac{1+z}{1-z} .
$$

Then

$$
\frac{z \mathcal{F}^{\prime}(z)}{\mathcal{F}(z)} \prec 1+\sin (z)
$$

Theorem 2. Assume that

$$
|\alpha| \geq \frac{(A-B)(\gamma+1)}{(1-|B|) \cos (1)-(1+|B|)(1+\gamma)(1+\sin h(1))} .
$$


If

$$
1+\alpha f(z) \prec \frac{1+A z}{1+B z},
$$

then

$$
\frac{\mathcal{F}(z)}{z} \prec 1+\sin (z),
$$

where $\mathcal{F}$ is the Bernardi integral operator defined in (1.3).

Proof. Let a function $w$ be defined by

$$
w(z)=\arcsin \left(\frac{\mathcal{F}(z)}{z}-1\right),
$$

where we have chosen the principle branches of the square root and logarithmic functions. Then $w$ is analytic in $\mathcal{D}$ with $w(0)=0$. We need only to show that $|w(z)|<1$ in $\mathcal{D}$. From (2.9), we have

$$
\frac{\mathcal{F}(z)}{z}=1+\sin (w(z)) .
$$

Also we define a function

$$
p(z)=1+\alpha f(z),
$$

where $p$ is analytic in $\mathcal{D}$ with $p(0)=1$. Now by using (2.10), (2.4) and (2.11), we have

$$
\left|\frac{p(z)-1}{A-B p(z)}\right|=\left|\frac{\alpha z\left[z w^{\prime}(z) \cos (w(z))+(1+\gamma)(1+\sin (w(z)))\right]}{(A-B)(1+\gamma)+\alpha B z\left[z w^{\prime}(z) \cos (w(z))+(1+\gamma)(1+\sin (w(z)))\right]}\right| .
$$

Suppose that there exists a point $z_{0} \in \mathcal{D}$ such that

$$
\max _{|z| \leq\left|z_{0}\right|}|w(z)|=\left|w\left(z_{0}\right)\right|=1 .
$$

By using Lemma 1 , there exists a number $m \geq 1$ such that $z_{0} w^{\prime}\left(z_{0}\right)=m w\left(z_{0}\right)$. We also suppose that $w\left(z_{0}\right)=e^{i \theta}$. Then we have

$$
\begin{aligned}
\left|\frac{p\left(z_{0}\right)-1}{A-B p\left(z_{0}\right)}\right| & =\frac{\alpha z_{0}\left[m e^{i \theta} \cos \left(e^{i \theta}\right)+(1+\gamma)\left(1+\sin \left(e^{i \theta}\right)\right)\right]}{(A-B)(1+\gamma)+\alpha B z_{0}\left[m e^{i \theta} \cos \left(e^{i \theta}\right)+(1+\gamma)\left(1+\sin \left(e^{i \theta}\right)\right)\right]} \\
& \geq \frac{|\alpha|\left[\left|m e^{i \theta} \cos \left(e^{i \theta}\right)\right|-\left|(1+\gamma)\left(1+\sin \left(e^{i \theta}\right)\right)\right|\right]}{(A-B)|(1+\gamma)|+|\alpha B|\left[\left|m e^{i \theta} \cos \left(e^{i \theta}\right)\right|+\left|(1+\gamma)\left(1+\sin \left(e^{i \theta}\right)\right)\right|\right]} \\
& \geq \frac{|\alpha|[m \cos (1)-(1+\gamma)(1+\sin h(1))]}{(A-B)(1+\gamma)+|\alpha B|[m \cos (1)+(1+\gamma)(1+\sin h(1))]} .
\end{aligned}
$$

Now let

$$
\Theta(m)=\frac{|\alpha|[m \cos (1)-(1+\gamma)(1+\sin h(1))]}{(A-B)(1+\gamma)+|\alpha B|[m \cos 1+(1+\gamma)(1+\sin h(1))]} .
$$


Then

$$
\Theta^{\prime}(m)=\frac{((A-B)+2|\alpha||B|(1+\sin h(1)))|\alpha|(\gamma+1) \cos (1)}{\{(A-B)(1+\gamma)+|\alpha B|[m \cos (1)+(1+\gamma)(1+\sin h(1))]\}^{2}}>0,
$$

which shows that $\Theta$ is an increasing function and it has its minimum value at $m=1$, so

$$
\left|\frac{p\left(z_{0}\right)-1}{A-B p\left(z_{0}\right)}\right| \geq \frac{|\alpha|[\cos (1)-(1+\gamma)(1+\sin h(1))]}{(A-B)(1+\gamma)+|\alpha B|[\cos (1)+(1+\gamma)(1+\sin h(1))]} .
$$

Now by $(2.7)$, we have

a contradiction to the hypothesis. Hence

$$
\left|\frac{p\left(z_{0}\right)-1}{A-B p\left(z_{0}\right)}\right| \geq 1
$$

$$
1+\alpha f(z) \prec \frac{1+A z}{1+B z} .
$$

By choosing $A=1, B=0$, we have the following result.

$$
\text { Corollary 3. Let }|\alpha| \geq \frac{(\gamma+1)}{\cos (1)-(1+|B|)(1+\gamma)(1+\sin h(1))} \text { and }
$$

Then

$$
\frac{\mathcal{F}(z)}{z} \prec 1+\sin (z) .
$$

By choosing $A=1, B=-1$, we have the following result.

Corollary 4. Let $|\alpha| \geq \frac{-1}{1+\sin h(1)}$ and

Then

$$
1+\alpha f(z) \prec \frac{1+z}{1-z} .
$$

$$
\frac{\mathcal{F}(z)}{z} \prec 1+\sin (z)
$$

Theorem 3. Assume that

$$
|\alpha| \geq \frac{(A-B)(\gamma+1)}{(1-|B|) \cos (1)-(1+|B|)(1+\gamma)(1+\sin h(1))} .
$$

If

then

$$
1+\alpha z f^{\prime}(z) \prec \frac{1+A z}{1+B z},
$$

$$
\mathcal{F}^{\prime}(z) \prec 1+\sin (z)
$$


where $\mathcal{F}$ is the Bernardi integral operator defined in (1.3).

Proof. Let a function $w$ be defined by

$$
w(z)=\arcsin \left(\mathcal{F}^{\prime}(z)-1\right),
$$

where we have chosen the principle branches of the square root and logarithmic functions. It is clear that $w$ is an analytic function in $\mathcal{D}$ with $w(0)=0$. We need only to show that $|w(z)|<1$ in $\mathcal{D}$. From (2.14), we have

$$
\mathcal{F}^{\prime}(z)=1+\sin (w(z)) .
$$

Differentiating (2.4), we get

$$
f^{\prime}(z)=\mathcal{F}^{\prime}(z)+\frac{z \mathcal{F}^{\prime \prime}(z)}{\gamma+1}
$$

Also we define a function

$$
p(z)=1+\alpha z f^{\prime}(z),
$$

where $p$ is analytic with $p(0)=1$. Now by using (2.15), (2.16) and (2.17), we have $\left|\frac{p(z)-1}{A-B p(z)}\right|=\left|\frac{\alpha z\left[z w^{\prime}(z) \cos (w(z))+(1+\gamma)(1+\sin (w(z)))\right]}{(A-B)(1+\gamma)+\alpha B z\left[z w^{\prime}(z) \cos (w(z))+(1+\gamma)(1+\sin (w(z)))\right]}\right|$.

Suppose that there exists a point $z_{0} \in \mathcal{D}$ such that

$$
\max _{|z| \leq\left|z_{0}\right|}|w(z)|=\left|w\left(z_{0}\right)\right|=1 \text {. }
$$

By using Lemma 1 , there exists a number $m \geq 1$ such that $z_{0} w^{\prime}\left(z_{0}\right)=m w\left(z_{0}\right)$. We also suppose that $w\left(z_{0}\right)=e^{i \theta}$. Then we have

$$
\begin{aligned}
\left|\frac{p\left(z_{0}\right)-1}{A-B p\left(z_{0}\right)}\right| & =\left|\frac{\alpha z_{0}\left[m e^{i \theta} \cos \left(e^{i \theta}\right)+(1+\gamma)\left(1+\sin \left(e^{i \theta}\right)\right)\right]}{(A-B)(1+\gamma)+\alpha B z_{0}\left[m e^{i \theta} \cos \left(e^{i \theta}\right)+(1+\gamma)\left(1+\sin \left(e^{i \theta}\right)\right)\right]}\right| \\
& \geq \frac{|\alpha|\left[\left|m e^{i \theta} \cos \left(e^{i \theta}\right)\right|-\left|(1+\gamma)\left(1+\sin \left(e^{i \theta}\right)\right)\right|\right]}{(A-B)|(1+\gamma)|+|\alpha B|\left[\left|m e^{i \theta} \cos \left(e^{i \theta}\right)\right|+\left|(1+\gamma)\left(1+\sin \left(e^{i \theta}\right)\right)\right|\right]} \\
& \geq \frac{|\alpha|[m \cos (1)-(1+\gamma)(1+\sin h(1))]}{(A-B)(1+\gamma)+|\alpha B|[m \cos (1)+(1+\gamma)(1+\sin h(1))]} .
\end{aligned}
$$

Now let

$$
\Xi(m)=\frac{|\alpha|[m \cos (1)-(1+\gamma)(1+\sin h(1))]}{(A-B)(1+\gamma)+|\alpha B|[m \cos (1)+(1+\gamma)(1+\sin h(1))]} .
$$

Then

$$
\Xi^{\prime}(m)=\frac{((A-B)+2|\alpha||B|(1+\sin h(1)))|\alpha|(\gamma+1) \cos (1)}{\{(A-B)(1+\gamma)+|\alpha B|[m \cos (1)+(1+\gamma)(1+\sin h(1))]\}^{2}}>0,
$$


which shows that $\Xi$ is an increasing function and it has its minimum value at $m=1$ so

$$
\left|\frac{p\left(z_{0}\right)-1}{A-B p\left(z_{0}\right)}\right| \geq \frac{|\alpha|[\cos (1)-(1+\gamma)(1+\sin h(1))]}{(A-B)(1+\gamma)+|\alpha B|[\cos (1)+(1+\gamma)(1+\sin h(1))]} .
$$

Now by (2.12), we have

a contradiction to the hypothesis

$$
\left|\frac{p\left(z_{0}\right)-1}{A-B p\left(z_{0}\right)}\right| \geq 1
$$

$$
1+\alpha z f^{\prime}(z) \prec \frac{1+A z}{1+B z} .
$$

Hence we have the required result.

By choosing $A=1, B=0$, we have the following result.

Corollary 5. Assume that

$$
|\alpha| \geq \frac{(\gamma+1)}{\cos (1)-(1+\gamma)(1+\sin h(1))} .
$$

If

$$
1+\alpha z f^{\prime}(z) \prec 1+z,
$$

then

$$
\mathcal{F}^{\prime}(z) \prec 1+\sin (z)
$$

By choosing $A=1, B=-1$, we have the following result.

Corollary 6. Assume that $|\alpha| \geq \frac{-1}{(1+\sin h(1))}$ and if

$$
1+\alpha z f^{\prime}(z) \prec \frac{1+z}{1-z},
$$

then

$$
\mathcal{F}^{\prime}(z) \prec 1+\sin (z) .
$$

3. BERNARDI'S INTEGRAL OPERATOR ASSOCIATED WITH COSINE FUNCTION

Theorem 4. Assume that

$$
|\alpha| \geq \frac{(A-B)(\gamma+1)}{(1-|B|) \sin (1)-(1+|B|)(\cosh (1)+\gamma) \cosh (1)} .
$$

If

$$
1+\alpha z\left(\frac{z f^{\prime}(z)}{f(z)}\right) \prec \frac{1+A z}{1+B z},
$$

then

$$
\frac{z \mathcal{F}^{\prime}(z)}{\mathcal{F}(z)} \prec \cos (z)
$$


where $\mathcal{F}$ is the Bernardi integral operator defined in (1.3).

Proof. Let a function $w$ be defined by

$$
w(z)=\cos ^{-1}\left(\frac{z \mathcal{F}^{\prime}(z)}{\mathcal{F}(z)}\right) .
$$

where we have chosen the principle branches of the square root and logarithmic functions. Since arccos function is defined as

$$
\arccos z=-i \log \left[z+i\left(1-z^{2}\right)^{1 / 2}\right],
$$

therefore $w$ is an analytic function in $\mathcal{D}$ with $w(0)=0$. To prove our result, we need only to show that $|w(z)|<1$ in $\mathcal{D}$. From (3.3), we have

$$
\frac{z \mathcal{F}^{\prime}(z)}{\mathcal{F}(z)}=\cos (w(z)) \text {. }
$$

Logarithmic differentiation of above relation yields

$$
1+\frac{z \mathcal{F}^{\prime \prime}(z)}{\mathcal{F}^{\prime}(z)}-\frac{z \mathcal{F}^{\prime}(z)}{\mathcal{F}(z)}=\frac{-z w^{\prime}(z) \sin (w(z))}{\cos (w(z))} .
$$

Using (1.3), we have

$$
(\gamma+1) f(z)=z \mathcal{F}^{\prime}(z)+\gamma \mathcal{F}(z) .
$$

Differentiating logarithmically, we have

$$
\begin{aligned}
\frac{z f^{\prime}(z)}{f(z)} & =\frac{z \mathcal{F}^{\prime}(z)}{\mathcal{F}(z)}\left\{\frac{1+\frac{z \mathcal{F}^{\prime \prime}(z)}{\mathcal{F}(z)}-\frac{z \mathcal{F}^{\prime}(z)}{\mathcal{F}(z)}}{\frac{z \mathcal{F}^{\prime}(z)}{\mathcal{F}(z)}+\gamma}+1\right\} \\
& =\frac{-z w^{\prime}(z) \sin (w(z))+(\cos (w(z))+\gamma) \cos (w(z))}{\cos (w(z))+\gamma} .
\end{aligned}
$$

Now we define a function

$$
p(z)=1+\alpha z\left(\frac{z f^{\prime}(z)}{f(z)}\right),
$$

where $p$ is analytic in $\mathcal{D}$ with $p(0)=1$. Also it is easy to see that

$$
\begin{aligned}
& \left|\frac{p(z)-1}{A-B p(z)}\right|= \\
& \left|\frac{\alpha z\left[-z w^{\prime}(z) \sin (w(z))+(\cos (w(z))+\gamma)(\cos (w(z)))\right]}{(A-B)(\cos (w(z))+\gamma)+\alpha B z\left[-z w^{\prime}(z) \sin (w(z))+(\cos (w(z))+\gamma)(\cos (w(z)))\right]}\right| .
\end{aligned}
$$

Suppose that there exists a point $z_{0} \in \mathcal{D}$ such that

$$
\max _{|z| \leq\left|z_{0}\right|}|w(z)|=\left|w\left(z_{0}\right)\right|=1 .
$$


By using Lemma 1 , there exists a number $m \geq 1$ such that $z_{0} w^{\prime}\left(z_{0}\right)=m w\left(z_{0}\right)$. We also suppose that $w\left(z_{0}\right)=e^{i \theta}$ and using (2.6), we have

$$
\begin{aligned}
& \left|\frac{p\left(z_{0}\right)-1}{A-B p\left(z_{0}\right)}\right|= \\
& \left|\frac{\alpha z_{0}\left[-z_{0} w^{\prime}\left(z_{0}\right) \sin \left(w\left(z_{0}\right)\right)+\left(\cos \left(w\left(z_{0}\right)\right)+\gamma\right)\left(\cos \left(w\left(z_{0}\right)\right)\right)\right]}{(A-B)\left(\cos \left(w\left(z_{0}\right)\right)+\gamma\right)+\alpha B z_{0}\left[-z_{0} w^{\prime}\left(z_{0}\right) \sin \left(w\left(z_{0}\right)\right)+\left(\cos \left(w\left(z_{0}\right)\right)+\gamma\right)\left(\cos \left(w\left(z_{0}\right)\right)\right)\right]}\right| \\
& =\left|\frac{\alpha z_{0}\left[-m e^{i \theta} \sin \left(e^{i \theta}\right)+\left(\cos \left(e^{i \theta}\right)+\gamma\right)\left(\cos \left(e^{i \theta}\right)\right)\right]}{(A-B)\left(\cos \left(e^{i \theta}\right)+\gamma\right)+\alpha B z_{0}\left[-m e^{i \theta} \sin \left(e^{i \theta}\right)+\left(\cos \left(e^{i \theta}\right)+\gamma\right) \cos \left(e^{i \theta}\right)\right]}\right| \\
& \geq \frac{\alpha\left[\left|m e^{i \theta} \sin \left(e^{i \theta}\right)\right|-\left|\left(\cos \left(e^{i \theta}\right)+\gamma\right) \cos \left(e^{i \theta}\right)\right|\right]}{(A-B)\left(\cos \left(e^{i \theta}\right)+\gamma\right)+|\alpha B|\left[\left|m\left(e^{i \theta}\right) \sin \left(e^{i \theta}\right)\right|+\left|\left(\cos \left(e^{i \theta}\right)+\gamma\right) \cos \left(e^{i \theta}\right)\right|\right]}
\end{aligned}
$$

Now let

$$
\Phi_{1}(m)=\frac{\alpha[m \sin (1)-(\cosh (1)+\gamma) \cosh (1)]}{(A-B)(\cosh (1)+\gamma)+\alpha B[m \sin (1)+(\cosh (1)+\gamma) \cosh (1)]} .
$$

Then

$$
\Phi_{1}^{\prime}(m)=\frac{|\alpha| \sin (1)(A-B)(\cosh (1)+\gamma)+2|\alpha|^{2}|B|(\cosh (1)+\gamma) \cosh (1)}{\{(A-B)(\cosh (1)+\gamma)+|\alpha||B|[m \sin (1)+(\cosh (1)+\gamma) \cosh (1)]\}^{2}}>0,
$$

which shows that $\Phi_{1}$ is an increasing function and has its minimum value at $m=1$ so

$$
\left|\frac{p\left(z_{0}\right)-1}{A-B p\left(z_{0}\right)}\right| \geq \frac{|\alpha|[\sin (1)-(\cosh (1)+\gamma) \cosh (1)]}{(A-B)(\cosh (1)+\gamma)+|\alpha||B|[\sin (1)+(\cosh (1)+\gamma) \cosh (1)]} .
$$

Now by (3.1), we have

$$
\left|\frac{p\left(z_{0}\right)-1}{A-B p\left(z_{0}\right)}\right| \geq 1
$$

a contradiction to the hypothesis. Hence the result is complete.

By choosing $A=1, B=0$, we have the following result.

Corollary 7. Assume that

$$
|\alpha| \geq \frac{(\gamma+1)}{\sin (1)-(\cosh (1)+\gamma) \cosh (1)} .
$$

If

$$
1+\alpha z\left(\frac{z f^{\prime}(z)}{f(z)}\right) \prec 1+z
$$

then

$$
\frac{z \mathcal{F}^{\prime}(z)}{\mathcal{F}(z)} \prec \cos (z)
$$


where $\mathcal{F}$ is the Bernardi integral operator defined in (1.3).

By choosing $A=1, B=-1$, we have the following result.

Corollary 8. Assume that

$$
|\alpha| \geq \frac{-(\gamma+1)}{(\cosh (1)+\gamma) \cosh (1)}
$$

If

$$
1+\alpha z\left(\frac{z f^{\prime}(z)}{f(z)}\right) \prec \frac{1+z}{1-z},
$$

then

$$
\frac{z \mathcal{F}^{\prime}(z)}{\mathcal{F}(z)} \prec \cos (z) \text {. }
$$

Theorem 5. Assume that

$$
\alpha \geq \frac{(A-B)(\gamma+1)}{(1-|B|) \sin (1)-(1+|B|)(\cosh (1)+\gamma) \cosh (1)} .
$$

If

$$
1+\alpha f(z) \prec \frac{1+A z}{1+B z},
$$

then

$$
\frac{\mathcal{F}(z)}{z} \prec \cos (z),
$$

where $\mathcal{F}$ is the Bernardi integral operator defined in (1.3).

Proof. Let a function $w$ be defined by

$$
w(z)=\arccos \left(\frac{\mathcal{F}(z)}{z}\right),
$$

where we have chosen the principle branches of the square root and logarithmic functions. Therefore $w$ is an analytic function in $\mathcal{D}$ with $w(0)=0$. We need only to show that $|w(z)|<1$ in $\mathcal{D}$. From (3.6), we have

$$
\frac{\mathcal{F}(z)}{z}=\cos w(z)
$$

Also we define a function

$$
p(z)=1+\alpha f(z),
$$

where $p$ is analytic in $\mathcal{D}$ with $p(0)=1$. Now by using (3.7), (2.4) and (3.8) we have

$$
\left|\frac{p(z)-1}{A-B p(z)}\right|=\left|\frac{\alpha\left[-z^{2} w^{\prime}(z) \sin (w(z))+z(1+\gamma) \cos (w(z))\right]}{(A-B)(1+\gamma)+\alpha B\left[-z^{2} w^{\prime}(z) \sin (w(z))+z(1+\gamma) \cos (w(z))\right]}\right| \text {. }
$$

Suppose that there exists a point $z_{0} \in \mathcal{D}$ such that

$$
\max _{|z| \leq\left|z_{0}\right|}|w(z)|=\left|w\left(z_{0}\right)\right|=1 .
$$


By using Lemma 1 , there exists a number $m \geq 1$ such that $z_{0} w^{\prime}\left(z_{0}\right)=m w\left(z_{0}\right)$. We also suppose that $w\left(z_{0}\right)=e^{i \theta}$ and by using (2.6), we have

$$
\begin{aligned}
\left|\frac{p\left(z_{0}\right)-1}{A-B p\left(z_{0}\right)}\right| & =\left|\frac{\alpha\left[-z_{0} m e^{i \theta} \sin \left(e^{i \theta}\right)+z_{0}(1+\gamma) \cos \left(e^{i \theta}\right)\right]}{(A-B)(1+\gamma)+\alpha B\left[-z_{0} m e^{i \theta} \sin \left(e^{i \theta}\right)+z_{0}(1+\gamma) \cos \left(e^{i \theta}\right)\right]}\right| \\
& \geq \frac{|\alpha|\left[\left|m e^{i \theta} \sin \left(e^{i \theta}\right)\right|-\left|(1+\gamma) \cos \left(e^{i \theta}\right)\right|\right]}{(A-B)|(1+\gamma)|+|\alpha B|\left[\left|m e^{i \theta} \sin \left(e^{i \theta}\right)\right|+\left|(1+\gamma) \cos \left(e^{i \theta}\right)\right|\right]} \\
& \geq \frac{|\alpha|[m \sin (1)-(1+\gamma) \cosh (1)]}{(A-B)(1+\gamma)+|\alpha B|[m \sin (1)+(1+\gamma) \cosh (1)]} .
\end{aligned}
$$

Now let

Then

$$
\Theta_{1}(m)=\frac{|\alpha|[m \sin 1-(1+\gamma) \cosh 1]}{(A-B)(1+\gamma)+|\alpha B|[m \sin 1+(1+\gamma) \cosh 1]} .
$$

$$
\Theta_{1}^{\prime}(m)=\frac{(A-B)(1+\gamma) \sin (1)+2|\alpha|^{2}|B|(1+\gamma) \cosh (1) \sin (1)}{\{(A-B)(1+\gamma)+|\alpha B|[m \sin (1)+(1+\gamma) \cosh (1)]\}^{2}}>0
$$

which shows that $\Theta_{1}$ is an increasing function and has its minimum value at $m=1$ so

$$
\left|\frac{p\left(z_{0}\right)-1}{A-B p\left(z_{0}\right)}\right|=\frac{|\alpha|[\sin (1)-(1+\gamma) \cosh (1)]}{(A-B)(1+\gamma)+|\alpha B|[\sin (1)+(1+\gamma) \cosh (1)]} .
$$

Now by (3.4), we have

$$
\left|\frac{p\left(z_{0}\right)-1}{A-B p\left(z_{0}\right)}\right| \geq 1
$$

a contradiction to the hypothesis. Hence it completes the proof.

By choosing $A=1, B=0$, we have the following result.

Corollary 9. Assume that

$$
|\alpha| \geq \frac{(\gamma+1)}{\sin (1)-(\cosh (1)+\gamma) \cosh (1)} .
$$

If

$$
1+\alpha f(z) \prec 1+z,
$$

then

$$
\frac{\mathcal{F}(z)}{z} \prec \cos (z)
$$

By choosing $A=1, B=-1$, we have the following result.

Corollary 10. Assume that

$$
|\alpha| \geq \frac{-(\gamma+1)}{(\cosh (1)+\gamma) \cosh (1)} .
$$


If

then

$$
1+\alpha f(z) \prec \frac{1+z}{1-z},
$$

$$
\frac{\mathcal{F}(z)}{z} \prec \cos (z) \text {. }
$$

Theorem 6. Assume that

$$
|\alpha| \geq \frac{(A-B)(\gamma+1)}{(1-|B|) \sin (1)-(1+|B|)(1+\gamma) \cosh (1)} .
$$

If

$$
1+\alpha z f^{\prime}(z) \prec \frac{1+A z}{1+B z},
$$

then

$$
\mathcal{F}^{\prime}(z) \prec \cos (z),
$$

where $\mathcal{F}$ is the Bernardi integral operator defined in (1.3).

Proof. Let a function $w$ be defined by

$$
w(z)=\arccos \mathcal{F}^{\prime}(z),
$$

where we have chosen the principle branches of the square root and logarithmic functions. It is clear that $w$ is an analytic function in $\mathcal{D}$ with $w(0)=0$. We need only to show that $|w(z)|<1$ in $\mathcal{D}$. From (3.11), we have

$$
\mathcal{F}^{\prime}(z)=\cos (w(z)) .
$$

Differentiating (2.4), we get

$$
f^{\prime}(z)=\mathcal{F}^{\prime}(z)+\frac{z \mathcal{F}^{\prime \prime}(z)}{\gamma+1} .
$$

Also we define a function

$$
p(z)=1+\alpha z f^{\prime}(z),
$$

where $p$ is analytic and $p(0)=1$. Now by using (3.12), (3.13) and (3.14), we have

$$
\left|\frac{p(z)-1}{A-B p(z)}\right|=\left|\frac{\alpha z\left[-z w^{\prime}(z) \sin (w(z))+(\gamma+1) \cos (w(z))\right]}{(A-B)(1+\gamma)+\alpha B z\left[-z w^{\prime}(z) \sin (w(z))+(\gamma+1) \cos (w(z))\right]}\right| .
$$

Suppose that there exists a point $z_{0} \in \mathcal{D}$ such that

$$
\max _{|z| \leq\left|z_{0}\right|}|w(z)|=\left|w\left(z_{0}\right)\right|=1
$$

By using Lemma 1 , there exists a number $m \geq 1$ such that $z_{0} w^{\prime}\left(z_{0}\right)=m w\left(z_{0}\right)$. We also suppose that $w\left(z_{0}\right)=e^{i \theta}$. Then we have

$$
\left|\frac{p\left(z_{0}\right)-1}{A-B p\left(z_{0}\right)}\right|=\left|\frac{\alpha z_{0}\left[-m e^{i \theta} \sin \left(e^{i \theta}\right)+(\gamma+1) \cos \left(e^{i \theta}\right)\right]}{(A-B)(1+\gamma)+\alpha B z_{0}\left[-m e^{i \theta} \sin \left(e^{i \theta}\right)+(\gamma+1) \cos \left(e^{i \theta}\right)\right]}\right|
$$




$$
\begin{aligned}
& \geq \frac{|\alpha|\left[\left|m e^{i \theta} \sin \left(e^{i \theta}\right)\right|-\left|(\gamma+1) \cos \left(e^{i \theta}\right)\right|\right]}{(A-B)(1+\gamma)+|\alpha B|\left[\left|m e^{i \theta} \sin \left(e^{i \theta}\right)\right|+\left|(\gamma+1) \cos \left(e^{i \theta}\right)\right|\right]} \\
& \geq \frac{|\alpha|[m \sin (1)-(1+\gamma) \cosh (1)]}{(A-B)(1+\gamma)+|\alpha B|[m \sin (1)+(1+\gamma) \cosh (1)]} .
\end{aligned}
$$

Now let

$$
\Xi_{1}(m)=\frac{|\alpha|[m \sin (1)-(1+\gamma) \cosh (1)]}{(A-B)(1+\gamma)+|\alpha B|[m \sin [1]+(1+\gamma) \cosh (1)]} .
$$

Then

$$
\Xi_{1}^{\prime}(m)=\frac{(A-B)(1+\gamma)|\alpha| \sin (1)+2(1+\gamma)|\alpha|^{2}|B| \cosh (1) \sin (1)}{\{(A-B)(1+\gamma)+|\alpha B|[m \sin (1)+(1+\gamma) \cosh (1)]\}^{2}}>0,
$$

which shows that $\Xi_{1}$ is an increasing function and has its minimum value at $m=1$ so

$$
\left|\frac{p\left(z_{0}\right)-1}{A-B p\left(z_{0}\right)}\right| \geq \frac{|\alpha|[\sin (1)-(1+\gamma) \cosh (1)]}{(A-B)(1+\gamma)+|\alpha B|[\sin (1)+(1+\gamma) \cosh (1)]} .
$$

Now by (3.9), we have

$$
\left|\frac{p\left(z_{0}\right)-1}{A-B p\left(z_{0}\right)}\right| \geq 1
$$

a contradiction to the hypothesis. Hence we have the required result.

By choosing $A=1, B=0$, we have the following result.

Corollary 11. Assume that

$$
|\alpha| \geq \frac{(\gamma+1)}{\sin (1)-(1+\gamma) \cosh (1)} .
$$

If

$$
1+\alpha z f^{\prime}(z) \prec 1+z,
$$

then

$$
\mathcal{F}^{\prime}(z) \prec \cos (z) .
$$

By choosing $A=1, B=-1$, we have the following result.

Corollary 12. Assume that

$$
|\alpha| \geq \frac{-(\gamma+1)}{(1+\gamma) \cosh (1)}
$$

If

$$
1+\alpha z f^{\prime}(z) \prec \frac{1+z}{1-z},
$$

then

$$
\mathcal{F}^{\prime}(z) \prec \cos (z) \text {. }
$$




\section{REFERENCES}

[1] M. Arif, K. Ahmad, J. L. Liu, and J. Sokól, "A new class of analytic functions associated with Salagean operator," J. Func. Spaces, vol. 2019, pp. 1-8, 2019, doi: 10.1155/2019/6157394.

[2] M. Arif, H. M. Srivastava, and S. Umar, "Some applications of a $q$-analogue of the Ruscheweyh type operator for multivalent functions," RACSAM, vol. 113, pp. 1211-1221, 2019, doi: 10.1007/s13398-018-0539-3.

[3] K. Bano and M. Raza, "Starlike functions associated with cosine functions," Bull. Iran. Math. Soc., vol. communicated, 2020, doi: 10.1007/s41980-020-00456-9.

[4] S. D. Bernardi, "Convex and starlike univalent functions," Trans. Amer. Math. Soc., vol. 135, pp. 429-446, 1969, doi: 10.2307/1995025.

[5] N. E. Cho, S. Kumar, V. Kumar, V. Ravichandran, and H. M. Srivastava, "Starlike functions related to the Bell numbers," Symmetry, vol. 11(2), p. 219, 2019, doi: 10.3390/sym11020219.

[6] N. E. Cho, V. Kumar, S. Kumar, and V. Ravichandran, "Radius problems for starlike functions associated with the sine function," Bull. Iran. Math. Soc., vol. 45(1), pp. 213-232, 2019, doi: 10.1007/s41980-018-0127-5.

[7] J. Dziok, R. K. Raina, and J. Sokól, "On a class of starlike functions related to a shell-like curve connected with Fibonacci numbers," Math. Comp. Model., vol. 57, pp. 1203-1211, 2013, doi: 10.1016/j.mcm.2012.10.023.

[8] I. S. Jack, "Functions starlike and convex of order alpha," J. London Math. Soc., vol. 2, pp. 469474, 1971, doi: 10.1112/j1ms/s2-3.3.469.

[9] W. Janowski, "Extremal problems for a family of functions with positive real part and for some related families," Ann. Polon. Math., vol. 23, pp. 159-177, 1970, doi: 10.4064/ap-23-2-159-177.

[10] S. Kanas and D. Răducanu, "Some class of analytic functions related to conic domains," Math. Slovaca, vol. 64, pp. 1183-1196, 2014, doi: 10.2478/s12175-014-0268-9.

[11] R. Kargar, A. Ebadian, and J. Sokół, "Radius problems for some subclasses of analytic functions," Compl Anal. Oper. Theo., vol. 11, pp. 1639-1649, 2017, doi: 10.1007/s11785-016-0584-x.

[12] S. Kumar and V. Ravichandran, "A subclass of starlike functions associated with a rational function," Southeast Asian Bull. Math., vol. 40, pp. 199-212, 2016.

[13] S. Mahmood, I. Khan, H. M. Srivastava, and S. N. Malik, "Inclusion relations for certain families of integral operators associated with conic regions," J. Inequal Appl., vol. 2019, p. 59, 2019, doi: 10.1186/s13660-019-2015-9.

[14] S. Mahmood, N. Raza, E. S. A. AbuJarad, G. Srivastava, H. M. Srivastava, and S. N. Malik, "Geometric properties of certain classes of analytic functions associated with a $q$-integral operator," Symmetry, vol. 11, p. 719, 2019, doi: 10.3390/sym11050719.

[15] R. Mendiratta, S. Nagpal, and V. Ravichandran, "On a subclass of strongly starlike functions associated with exponential function," Bull. Malaysian Math. Sci. Soc., vol. 38, pp. 365-386, 2015, doi: 10.1007/s40840-014-0026-8.

[16] R. K. Raina and J. Sokół, "Some properties related to a certain class of starlike functions," C. $R$. Math., vol. 353, pp. 973-978, 2015, doi: 10.1016/j.crma.2015.09.011.

[17] K. Sharma, N. K. Jain, and V. Ravichandran, "Starlike functions associated with a cardioid," Afrika Math., vol. 27, pp. 923-939, 2016, doi: 10.1007/s13370-015-0387-7.

[18] L. Shi, I. Ali, M. Arif, N. E. Cho, S. Hussain, and H. Khan, "A study of third hankel determinant problem for certain subfamilies of analytic functions involving cardioid domain," Mathematics, vol. 7, p. 418, 2019, doi: 10.3390/math7050418.

[19] L. Shi, H. M. Srivastava, M. Arif, S. Hussain, and K. Hassan, "An investigation of the third Hankel determinant problem for certain subfamilies of univalent functions involving the exponential function," Symmetry, vol. 11, p. 598, 2019, doi: 10.3390/sym11050598. 
[20] Y. J. Sim, O. S. Kwon, N. E. Cho, and H. M. Srivastava, "Some classes of analytic functions associated with conic regions," Taiwanese J. Math., vol. 16, pp. 387-408, 2012.

[21] Y. Sim, O. Kwon, N. E. Cho, and H. M. Srivastava, "Bounds for the real parts and arguments of normalized analytic functions defined by the Srivastava-Attiya operator," J. Comput. Anal. Appl., vol. 28 , pp. 628-645, 2020.

[22] J. Sokól and J. Stankiewicz, "Radius of convexity of some subclasses of strongly starlike functions," Zeszyty Nauk. Politech. Rzeszowskiej Mat., vol. 19, pp. 101-105, 1996.

[23] H. M. Srivastava, "Operators of basic (or $q$-) calculus and fractional $q$-calculus and their applications in geometric function theory of complex analysis," Iran J. Sci. Technol. Trans. Sci., vol. 44, pp. 327-344, 2020, doi: 10.1007/s40995-019-00815-0.

[24] H. M. Srivastava, M. K. Aouf, and A. O. Mostafa, "Some properties of analytic functions associated with fractional $q$-calculus operators," Miskolc Math. Notes, vol. 20, pp. 1245-1260, 2019, doi: 10.18514/MMN.2019.3046.

[25] H. M. Srivastava, M. R. Khan, and M. Arif, "Some subclasses of close-to-convex mappings associated with conic regions," Appl. Math. Comput, vol. 285, pp. 94-102, 2016, doi: 10.1016/j.amc.2016.03.025.

[26] H. M. Srivastava, A. Motamednezhad, and E. A. Adegani, "Faber polynomial coefficient estimates for bi-univalent functions defined by using differential subordination and a certain fractional derivative operator," Mathematics, vol. 8, p. 172, 2020, doi: 10.3390/math8020172.

Authors' addresses

Muhammad Arif

Department of Mathematics, Abdul Wali Khan University, Mardan, Pakistan

E-mail address: marifmaths@awkum.edu.pk

\section{Mohsan Raza}

Department of Mathematics, Government College University, Faisalabad, Pakistan

E-mail address: mohsan976@yahoo.com

Miraj Ul Haq

Department of Mathematics, Abdul Wali Khan University, Mardan, Pakistan

E-mail address: merajkhan054@gmail.com

\section{Gautam Srivastava}

Department of Mathematics and Computer Science, Brandon University, Brandon, Canada Research Center for Interneural Computing, China Medical University, Taichung, Taiwan, Republic of China

E-mail address: SRIVASTAVAG@brandonu.ca 